\title{
Muon $(g-2)$ in the Standard Model and supersymmetric extensions
}

\author{
Markus Bach ${ }^{1}$, Dominik Stöckinger ${ }^{1}$, Hyejung Stöckinger-Kim ${ }^{1}$, and Jae-hyeon Park ${ }^{2}$ \\ ${ }^{1}$ Institut für Kern- und Teilchenphysik, Technische Universität Dresden, Dresden, Germany \\ ${ }^{2}$ Departament de Física Teòrica and IFIC, Universitat de València-CSIC, 46100, Burjassot, Spain
}

\begin{abstract}
Recent new results on the SM and the SUSY prediction for the muon $(g-2)$ are briefly reviewed, and a SUSY scenario with particularly large contributions is discussed.
\end{abstract}

\section{Introduction}

In spite of the discovery of a Higgs boson, the first run of the LHC has not revealed any significant sign for physics beyond the Standard Model (SM). On the other hand, in spite of tremendous scrutiny in the past years, the gap between the SM-prediction and the experimental value of the muon anomalous magnetic moment has not been reduced. As discussed in many other talks at this meeting, recent progress on the hadronic contributions to $(g-2)_{\mu}$ confirm a deviation of more than 3 standard deviations or approximately $3 \times 10^{-9}$. Hence the question remains whether this deviation is due to physics beyond the SM.

In the following we first explain the key ingredients of the more precise evaluation of the electroweak SM contributions in Ref. [1]. Then we argue that precise predictions are also necessary in models different from the SM, and we discuss the status of the prediction in the minimal supersymmetric standard model (MSSM). Finally we show that within the framework of the MSSM, very large contributions to $(g-2)_{\mu}$ are possible even if the masses of all new particles are in the $\mathrm{TeV}$ region.

\section{The electroweak SM contribution}

The electroweak contributions to the SM prediction for $(g-2)_{\mu}$ have been computed for the first time at the twoloop level in Refs. [2, 3]. Two simplifications were used in these computations: (1) the fermion-loop contributions were evaluated using elementary light quarks. (2) the dependence on the Higgs boson mass was approximated by taking the Higgs boson mass either as equal to the topquark mass, or taking it to infinity or to zero.

Point (1) was improved in a series of papers which evaluated the hadronic contributions using hadronic models and non-perturbative information; the currently best result and a discussion of other references has been given in Ref. [4]. Ref. [4] also evaluated the leading three-loop logarithmic contributions.

Point (2) was improved in Refs. [5, 6], where in particular the bosonic electroweak two-loop contributions have been evaluated taking into account the exact Higgs boson mass dependence.

However, the results of these references were never combined consistently, because the employed renormalization schemes were different. Hence, in Ref. [1] the twoloop computation of the electroweak contributions has been repeated in the appropriate renormalization scheme, so that a combination with the hadronic and the leading three-loop results of Ref. [4] became possible. Fig. 1 shows sample Feynman diagrams and numerical results of the bosonic and the Higgs-dependent fermionic two-loop contributions.

Furthermore, the remaining sources of parametric and theory uncertainties have been analyzed. The final result obtained in Ref. [1] for the SM electroweak contributions is

$$
a_{\mu}^{\mathrm{EW}}=(153.6 \pm 1.0) \times 10^{-11} .
$$

Here the parametric uncertainty due to the input values of the Higgs boson, W- and Z-boson and top-quark is negligible; the uncertainty is dominated by the one of the hadronic contributions and has been taken over from Ref. [4].

\section{Contributions in supersymmetric models}

\subsection{Motivation}

Figure 2 provides motivation to consider $a_{\mu}$ as a constraint on the SUSY parameter space. In red it shows the prediction for $a_{\mu}^{\mathrm{SUSY}}$ for the SPS benchmark points [7]. As can be seen these well-motivated points lead to very different contributions, and independently of the resulting value, the future $a_{\mu}$ experiments will exclude most points. In green the figure shows "degenerate" points found in Ref. [8], which correspond to distinct SUSY parameter scenarios which however lead to the same LHC signatures. Combining LHC-measurements with $a_{\mu}$ will allow to select between these points. 

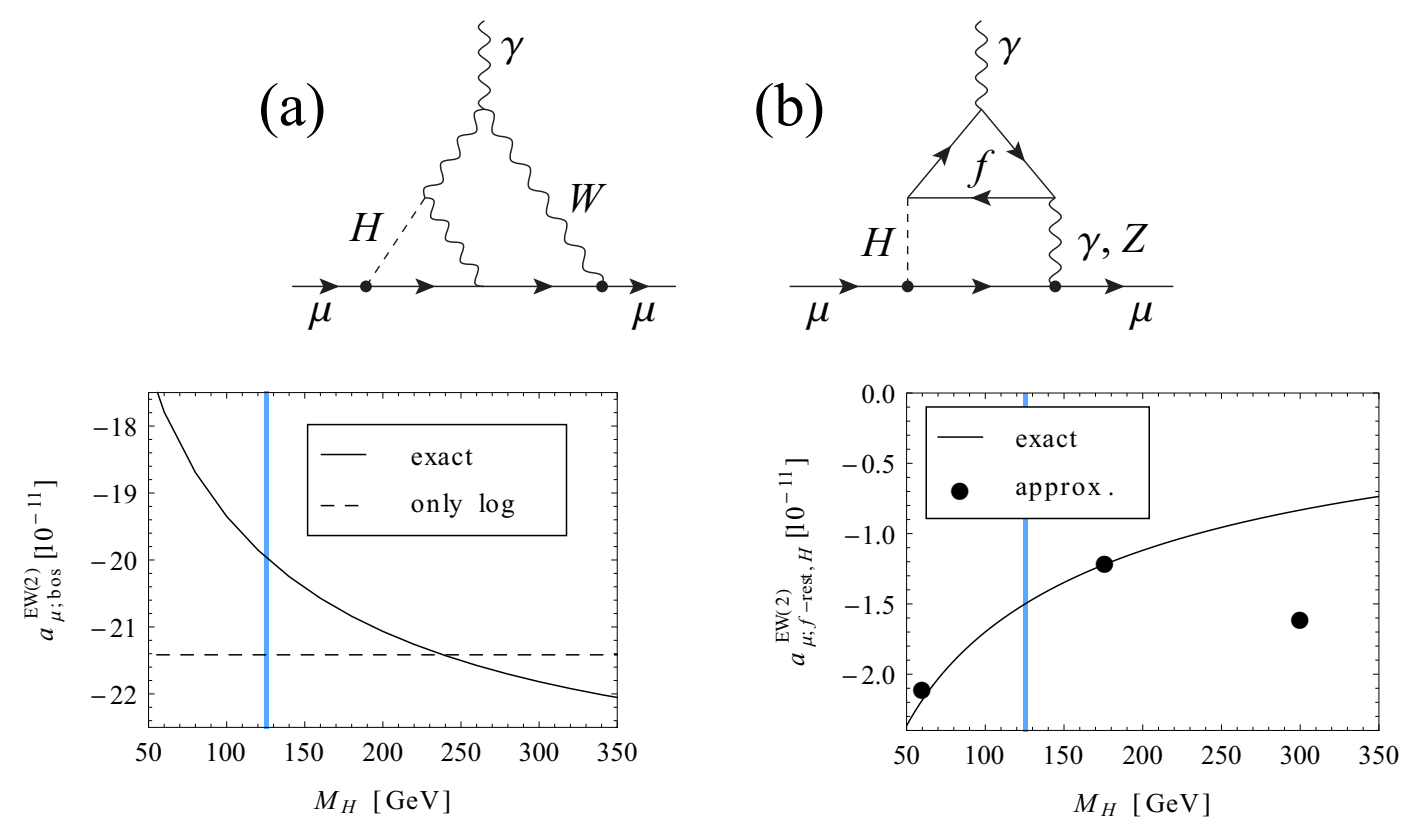

Figure 1. Sample Feynman diagrams and numerical results for the full bosonic electroweak two-loop contributions $a_{\mu \text {; bos }}^{\mathrm{EW}(2)}($ left $)$ and for the Higgs-dependent fermion-loop electroweak two-loop contributions $a_{\mu, \mathrm{f}-\text { rest, } \mathrm{EW}}^{\mathrm{E}(2)}$ (right), both as a function of the Higgs boson mass. The vertical band indicates the measured value of $M_{H}$. The dashed line in the left plot corresponds to the leading logarithmic approximation as defined in Ref. [5]. The fat dots in the right plot correspond to the approximations for $M_{H}=60 \mathrm{GeV}, m_{t}, 300 \mathrm{GeV}$ given in Ref. [2]. Figures from Ref. [1].

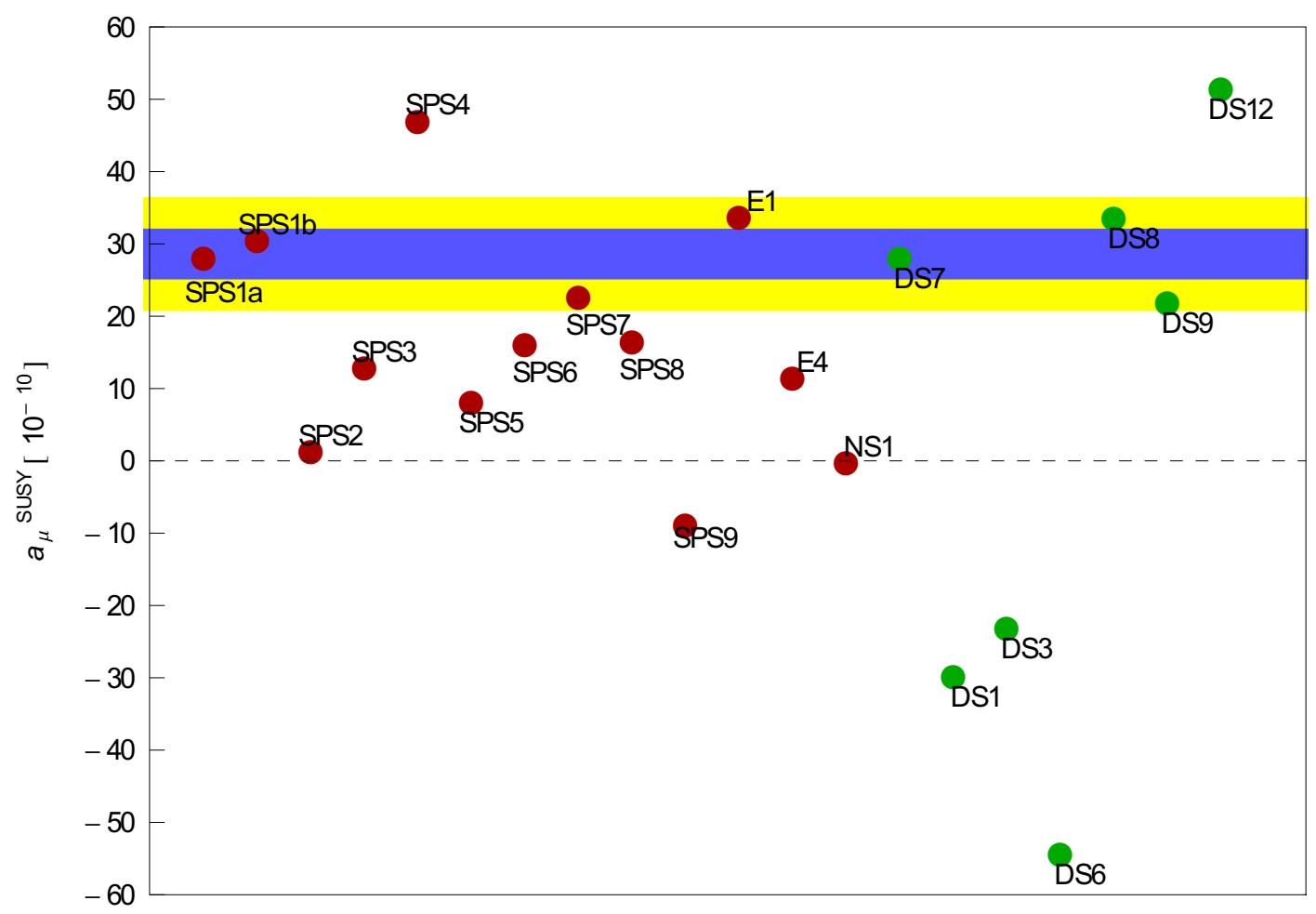

Figure 2. The prediction for $a_{\mu}^{\text {SUSY }}$ for the case of the SPS benchmark points [7] and for the "degenerate" points identified in Ref. [8]. The bands indicate the current deviation between experiment and the SM prediction, and the expected precision of future measurements, assuming the same central value. Figure from Ref. [9]. 


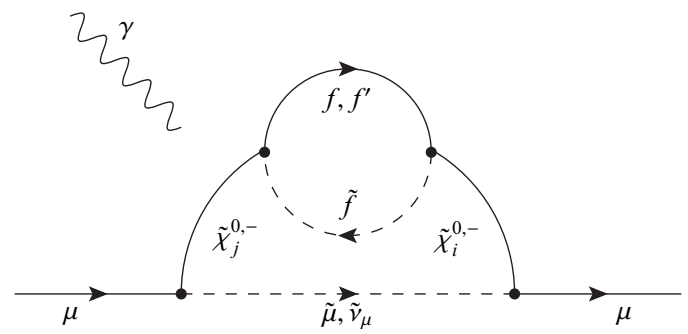

Figure 3. Sample two-loop diagram contributing to the fermion/sfermion-loop contributions of Refs. [12, 13].

\subsection{Precision prediction for $a_{\mu}^{\text {SUSY }}$}

In view of these prospects and of the expected precision of the future experiments, it is also motivated to aim for a SUSY prediction for $a_{\mu}$ with a theory uncertainty which is as small as possible, ideally smaller than the future experimental uncertainty. This requires the computation of the full two-loop contributions to $a_{\mu}^{\text {SUSY }}$, see Ref. [10].

This goal has not been reached, but significant progress has been achieved in the past years. A computer program has been published in Ref. [11], which implements the currently best prediction of $a_{\mu}^{\text {SUSY }}$, including all corrections discussed below.

Here we highlight in particular the computation of the fermion/sfermion-loop contributions in Refs. [12, 13]. A sample diagram is shown in Fig. 3. This computation has two interesting qualitative consequences. The first is that it reduces the theory uncertainty resulting from the possibility to choose different renormalization schemes for the finestructure constant $\alpha$ and the weak mixing angle $\sin \theta_{W}$. The one-loop contributions directly depend on these input parameters and thus on the scheme choice. The leading differences between the usually considered schemes originate from fermion or sfermion loops in gauge boson self energies. These terms are precisely included in the counterterm computation of Refs. [12, 13]. The sum of the one-loop contributions and the two-loop contributions of these references is essentially scheme independent (up to 3-loop effects and effects from non-fermion/sfermion-loop contributions).

A second qualitative outcome of that computation is that the contributions from fermion/sfermion loops involve potentially large logarithms between different SUSY particle masses. Particularly very heavy stops in the multi$\mathrm{TeV}$ region can lead to non-decoupling corrections of the SUSY one-loop contribution to $a_{\mu}$, which can amount to more than $10 \%$. These non-decoupling logarithms also allow to obtain a compact approximation of the fermion/sfermion-loop contributions.

\section{$3.3 a_{\mu}^{\mathrm{SUSY}}$ for $\tan \beta \rightarrow \infty$}

It is well known that the leading SUSY contributions to $a_{\mu}$ are proportional to $\tan \beta$. However, this proportionality is not exact - in fact for large $\tan \beta$ higher-order effects modify the behaviour, and the limit $\tan \beta \rightarrow \infty$ exists and is viable $[14,15]$, and it is intriguing to study how large $a_{\mu}$ can become in this limit. Here we briefly review the study of Ref. [16]. In order to understand the behaviour it is useful to write the muon mass and the muon magnetic moment as

$$
\begin{aligned}
m_{\mu} & =y_{\mu} v_{d}+y_{\mu} v_{u} \Delta_{\mu}^{\mathrm{red}}+\ldots, \\
a_{\mu}^{\mathrm{SUSY}} & =\frac{y_{\mu} v_{u} a_{\mu}^{\mathrm{red}}}{m_{\mu}}+\ldots,
\end{aligned}
$$

where $m_{\mu}$ and $y_{\mu}$ are the muon pole mass and Yukawa coupling, $v_{u, d}$ is the up/down-type Higgs vacuum expectation value, and the dots denote terms proportional to $v_{d}$ instead of $v_{u}$. The limit $\tan \beta \rightarrow \infty$ is equivalent to $v_{d} \rightarrow 0$, and in this limit we obtain

$$
a_{\mu}^{\mathrm{SUSY}}=\frac{a_{\mu}^{\mathrm{red}}}{\Delta_{\mu}^{\mathrm{red}}} .
$$

Here $a_{\mu}^{\text {red }}$ and $\Delta_{\mu}^{\text {red }}$ are given by calculable, finite loop diagrams, which depend (at the one-loop level) on the gaugino, Higgsino, and smuon mass parameters $M_{1,2}, \mu, m_{L, R}$.

Figure 4 shows the resulting $a_{\mu}^{\text {SUSY }}$ as a function of two mass ratios. The gray area shows the region with negative $a_{\mu}^{\text {SUSY }}$, which cannot explain the currently observed deviation between experiment and SM-prediction. The case with universal SUSY masses (all mass ratios equal to one) belongs to the region. However, if there are mass splittings (either Higgsino mass or left-handed smuon mass much larger than the other masses), the resulting $a_{\mu}^{\text {SUSY }}$ becomes positive.

Two sample parameter choices (with mass values given in $\mathrm{TeV}$ ) and the results for $a_{\mu}^{\mathrm{SUSY}}$ are as follows:

\begin{tabular}{ccccc|c}
\hline$\mu$ & $M_{1}$ & $M_{2}$ & $m_{L}$ & $m_{R}$ & $a_{\mu} / 10^{-9}$ \\
\hline 15 & 1 & -1 & 1 & 1 & 3.01 \\
1.3 & 1.3 & -1.3 & 26 & 1.3 & 2.90 \\
\hline
\end{tabular}

The table and the figure show that the limit $\tan \beta \rightarrow \infty$ provides a promising example of a scenario which could explain the deviation in $a_{\mu}$ even though all new particle masses are at or above the TeV-scale.

\section{References}

[1] C. Gnendiger, D. Stöckinger and H. Stöckinger-Kim, Phys. Rev. D 88 (2013) 053005.

[2] A. Czarnecki, B. Krause and W. J. Marciano, Phys. Rev. D 52 (1995) 2619.

[3] A. Czarnecki, B. Krause and W. J. Marciano, Phys. Rev. Lett. 76 (1996) 3267.

[4] A. Czarnecki, W. J. Marciano, A. Vainshtein Phys.Rev.D 67 (2003) 073006, Erratum-ibid.D73 (2006) 119901.

[5] S. Heinemeyer, D. Stöckinger and G. Weiglein, Nucl. Phys. B 699 (2004) 103.

[6] T. Gribouk and A. Czarnecki, Phys. Rev. D 72 (2005) 053016. 

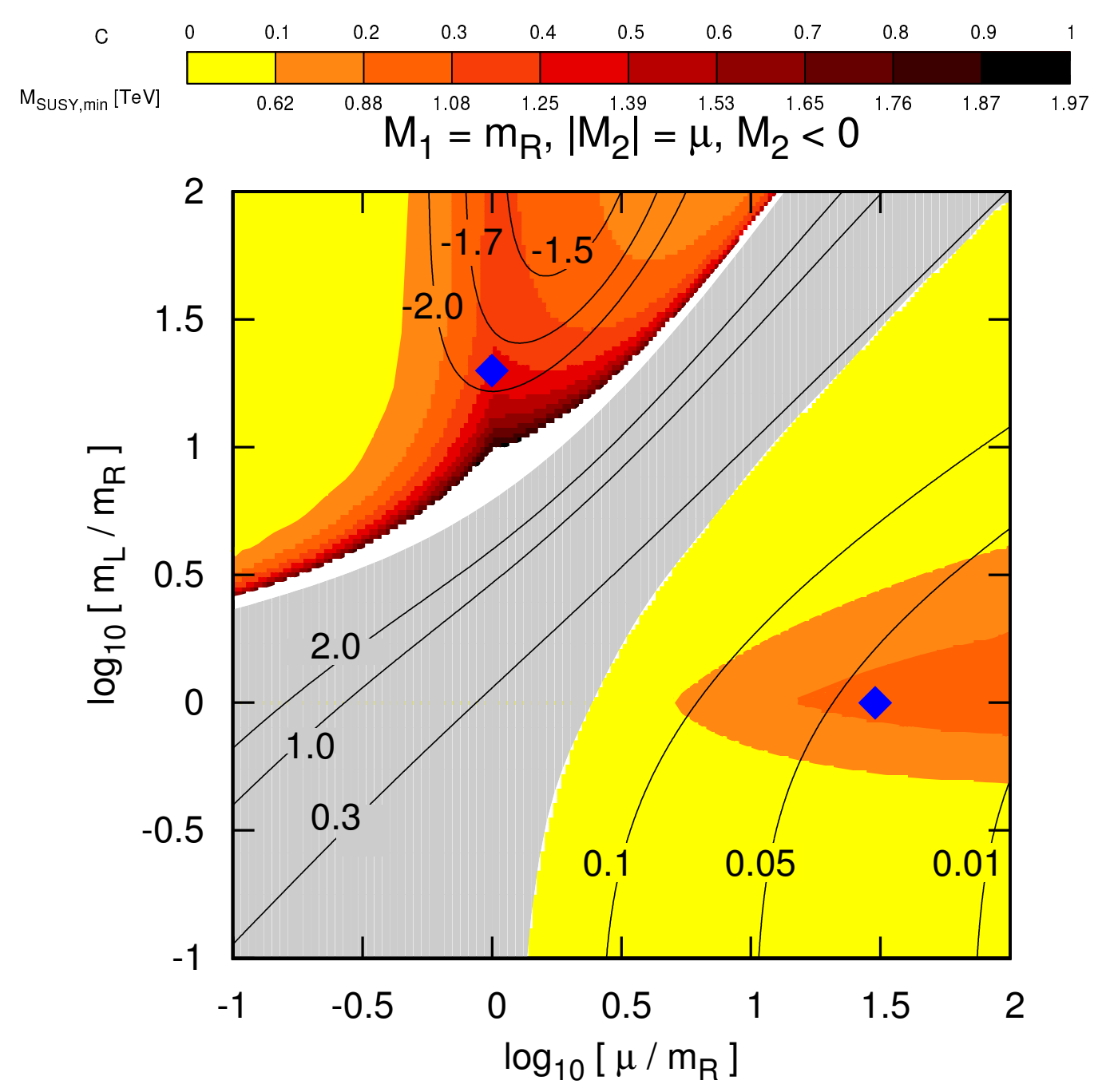

Figure 4. The minimum SUSY mass $M_{\mathrm{SUSY} \text {, } \min }=\min \left(M_{1},\left|M_{2}\right|, m_{L}, m_{R}, \mu\right)$ for which $a_{\mu}^{\mathrm{SUSY}}$ fully explains the current deviation between experiment and SM in the limit $\tan \beta \rightarrow \infty$. Equivalently the value $C$, defined by $a_{\mu}^{\text {SUSY }}=C m_{\mu}^{2} / M_{\mathrm{SUSY} \text { min }}^{2}$, is given. The black lines denote values of the muon Yukawa coupling, and the blue squares denote benchmark points. The figure has been taken from Ref. [16].

[7] B. C. Allanach et al., "The Snowmass points and slopes: Benchmarks for SUSY searches," in Proc. of the APS/DPF/DPB Summer Study on the Future of Particle Physics (Snowmass 2001) ed. N. Graf, Eur. Phys. J. C 25 (2002) 113 [eConf C010630 (2001) P125] [arXiv:hep-ph/0202233].

[8] C. Adam, J. -L. Kneur, R. Lafaye, T. Plehn, M. Rauch and D. Zerwas, Eur. Phys. J. C 71 (2011) 1520 [arXiv:1007.2190 [hep-ph]].

[9] J. P. Miller, E. de Rafael, B. L. Roberts and D. Stöckinger, Ann. Rev. Nucl. Part. Sci. 62 (2012) 237.

[10] D. Stöckinger, J. Phys. G G 34 (2007) R45.
[11] P. Athron et al., arXiv:1510.08071 [hep-ph].

[12] H. G. Fargnoli, C. Gnendiger, S. Paßehr, D. Stöckinger and H. Stöckinger-Kim, Phys. Lett. B 726 (2013) 717 [arXiv:1309.0980 [hep-ph]].

[13] H. G. Fargnoli, C. Gnendiger, S. Paßehr, D. Stöckinger and H. Stöckinger-Kim, JHEP 1402 (2014) 070 [arXiv:1311.1775 [hep-ph]].

[14] B. A. Dobrescu and P. J. Fox, Eur. Phys. J. C 70 (2010) 263 [arXiv:1001.3147 [hep-ph]].

[15] W. Altmannshofer and D. M. Straub, JHEP 1009 (2010) 078 [arXiv:1004.1993 [hep-ph]].

[16] M. Bach, J. h. Park, D. Stöckinger and H. StöckingerKim, arXiv:1504.05500 [hep-ph]. 